\title{
El reto de los profesores de secundaria ante las redes sociales
}

\author{
Isabel Dans Álvarez de Sotomayor \\ Eduardo José Fuentes Abeledo \\ Universidade de Santiago de Compostela. España. \\ isabel.dans@usc.es \\ eduardo.fuentes@usc.es
}

Mercedes González Sanmamed

Universidade da Coruña. España.

mercedes.gonzalez.sanmamed@udc.es

Pablo César Muñoz Carril

Universidade de Santiago de Compostela. España.

pablocesar.munoz@usc.es

Recibido: 5/12/19

Aceptado: $7 / 9 / 20$

Publicado: 29/1/2021

\section{Resumen}

En el contexto educativo, la integración de la tecnología genera posiciones ambivalentes: se percibe como una oportunidad para la innovación, pero también como un elemento disruptivo que suscita preocupación. Esta doble visión se pone de manifiesto cuando el profesorado se enfrenta al reto de las redes sociales y valora su utilidad en las aulas. El propósito de la investigación que presentamos ha sido analizar las percepciones del profesorado acerca del uso de las redes sociales por parte del alumnado de secundaria e identificar los requisitos materiales y formativos de los docentes para que puedan incorporarlas a su labor educativa. Se ha realizado un estudio cualitativo y la técnica utilizada ha sido la entrevista grupal. Los resultados muestran que los docentes consideran que el uso de las redes sociales en la enseñanza puede resultar motivador, pero alertan de los riesgos que puede acarrear entre los jóvenes. Las conclusiones se plantean a tres niveles: el profesorado está dispuesto a integrar estos recursos en el ámbito educativo, pero para ello se requiere un cambio a nivel profesional, sobre todo en lo relativo a una mejor formación en competencias digitales, y también reclama un aumento de los recursos disponibles en los centros docentes.

Palabras clave: redes sociales; profesorado; adolescencia; educación secundaria

Resum. El repte dels professors de secundària davant les xarxes socials

En el context educatiu, la integració de la tecnologia genera posicions ambivalents: es percep com una oportunitat per innovar, però també com un element disruptiu que suscita preocupació. Aquesta doble visió es posa de manifest quan el professorat s'enfronta al repte de les xarxes socials i en valora la utilitat a les aules. El propòsit d'aquesta recerca ha estat analitzar les percepcions del professorat sobre l'ús de les xarxes socials per part de l'alumnat de secundària i identificar els requisits materials i formatius dels docents perquè 
puguin incorporar-les a la seva tasca educativa. S'ha realitzat un estudi qualitatiu i la tècnica utilitzada ha estat l'entrevista grupal. Els resultats mostren que els docents consideren que l'ús de les xarxes socials en l'ensenyament pot resultar motivador, però alerten dels riscos que pot implicar entre els joves. Les conclusions es plantegen a tres nivells: el professorat està disposat a integrar aquests recursos en l'àmbit educatiu, però per a això es requereix un canvi a nivell professional, sobretot quant a una millor formació en competències digitals, i també reclama un augment dels recursos disponibles als centres docents.

Paraules clau: xarxes socials; professorat; adolescència; educació secundària

\section{Abstract. The challenge of secondary teachers in the light of the social networks}

In the educational context, the integration of technology generates ambivalent positions: it is perceived as an opportunity for innovation, but also as a disruptive element that raises concerns. This double vision is evident when teachers face the challenge of social networks and value their usefulness in classrooms. The purpose of this research has been to analyze the teachers' perceptions about the use of social networks by high school students and to identify the material and training requirements of teachers so that they can incorporate them into their educational work. A qualitative study has been carried out and the technique used has been the group interview. The results show that teachers consider that the use of social networks in teaching can be motivating, but they warn of the risks that they can entail for young people. The conclusions are proposed at three levels: teachers are willing to integrate these resources into the educational field, but this requires a change at the professional level, especially with regard to better training in digital skills; and they also demand an increase in the resources available in educational centers.

Keywords: social networks; teachers; teenagers; secondary school

\section{Sumario}
1. Presentación y justificación
3. Resultados del objeto de estudio
4. Discusión y conclusiones
2. Materiales y métodos
Referencias bibliográficas

\section{Presentación y justificación del objeto de estudio}

El interés científico por las redes sociales ha aumentado considerablemente en los últimos años en sintonía con su generalización entre la ciudadanía y, particularmente, entre los jóvenes, lo que ha motivado también un análisis de sus posibilidades educativas (Manca y Ranieri, 2015; Zulkanain, Miskon, Abdullah, Ali y Bahari, 2019). Lo cierto es que la mayoría de estos estudios se dirigen a la etapa universitaria, y los datos que ofrecen no siempre son comparables por la diversidad de las muestras. Cartagena (2016) propone el desarrollo de investigaciones cualitativas para dar cuenta de la complejidad del fenómeno. Un ejemplo es el estudio de López, Civís y Molina (2018) sobre las redes sociales en maestros noveles. 
Las oportunidades de uso educativo que brindan las redes sociales han sido descritas por diversos autores en distintos contextos (González, Muñoz y Dans, 2017; Junco, 2015), con lo que se elimina la separación existente entre el ya tradicional uso lúdico y socializador que caracteriza a los espacios juveniles y el uso productivo más recurrente en los adultos (Garrido, Busquet y Munté, 2016; Ballesteros y Picazo, 2018). Además, diversas investigaciones constatan que la incorporación de los medios sociales a las aulas de secundaria conlleva importantes beneficios (véanse los estudios de García-Martín y Cantón-Mayo, 2019; Kim, Holman y Goodreau, 2015; Hughes, Ko, Lim y Liu, 2015).

Frente a las ventajas también se alzan las limitaciones, por ejemplo, las recogidas por Jiménez, Garmendia y Casado (2018) o Smahel et al. (2020), quienes afirman cómo paradójicamente los beneficios para la mayoría (facilidad de acceso y ubicuidad) se pueden convertir en riesgos para las minorías. Junto a ello, los datos evidencian que no siempre existe correlación entre la competencia digital y la mejora del rendimiento académico (De Pablos Pons, Colás Bravo, Conde Jiménez y Reyes de Cózar, 2017; García-Martín y Cantón-Mayo, 2019; Fuentes, Albertos y Torrano, 2019).

Otra perspectiva deriva del amplio número de estudios sobre Facebook en el ámbito educativo que recogen Manca y Ranieri (2015). Tras reseñar las controversias más significativas, concluyen que dicha red social está siendo infrautilizada en educación. Para ello se requiere un giro metodológico, invertir en motivación y combatir los prejuicios culturales. También la prohibición del uso del móvil en los centros como herramienta de aprendizaje apunta a una cuestión altamente conflictiva que involucra a la Administración y a las familias (Pedro, Barbosa y Santos, 2018).

En concreto, las mejoras que pueden aportar las redes sociales en el ámbito escolar se pueden agrupar en dos grandes bloques: fomento de las competencias personales y promoción de las competencias para el aprendizaje. Entre las primeras, el desarrollo emocional de los estudiantes y el profesorado juega un papel relevante (Greenhow y Burton, 2011), ya que coadyuva a generar un buen clima en la comunidad educativa (Asterhan y Rosenberg, 2015). El empoderamiento de los jóvenes (Gamito, Aristizabal, Vizcarra y León, 2020) contribuye a aumentar su autoconcepto y la confianza en sus posibilidades. A esto se asocia el crecimiento paralelo del profesorado, con lo que se mejoran los procesos organizativos (Hughes et al., 2015) que contribuyen a facilitar una mayor integración de docentes y alumnado (Fentry, Boykin y Vickery, 2017). A través de las redes sociales se potencia el desarrollo profesional, en cuanto aumenta el capital social estructural, relacional y cognitivo (López et al., 2018). El juicio del docente sobre su propia capacidad para conseguir que los estudiantes alcancen resultados satisfactorios requiere condiciones de aprendizaje adecuadas, flexibilización en las estrategias y niveles altos de participación (Cartagena, 2016).

Los efectos también son positivos sobre las competencias para el aprendizaje, tales como la creatividad (Pedro, Barbosa y Santos, 2018), la colaboración (Muñoz, Dans y González, 2019), las habilidades de pensamiento (Plaza de 
la Hoz, 2018) y la comunicación interpersonal (Zulkanain et al., 2019). Existen cinco dimensiones pedagógicas vinculadas a la utilización de las redes sociales en el aula descritas por Said y Tahir (2013): participativa, interactiva, social, cognitiva y valorativa. Favorecer este tipo de aprendizaje pasa por nuevos diseños y adaptaciones metodológicas en los que la alfabetización digital del alumnado cobra especial relevancia (Topaloglu, Caldidi y Oge, 2016).

$\mathrm{El}$ agente fundamental del cambio formativo es el docente, en quien recae el peso del nuevo rol. El profesorado es consciente de su papel como mediador en el proceso educativo, pero un temor le atenaza: la mitificación de un adolescente supuestamente experto en tecnología (De Pablos Pons, Colás Bravo, Conde Jiménez y Reyes de Cózar, 2017).

El interés por las posibilidades educativas de las redes sociales en la secundaria constituye la principal motivación para el desarrollo de la investigación que presentamos, surgida en el contexto de un estudio más amplio realizado en la ciudad de A Coruña con estudiantes de cuarto de ESO, sus familias y sus profesores. Los agentes fundamentales del proceso educativo en la adolescencia - madres, padres y docentes - observan y utilizan las redes sociales de diferente modo y en torno a ellas mantienen preocupaciones y expectativas diversas. En cualquier caso, y a pesar de las posibles discrepancias, resulta insoslayable revisar la toma de decisiones sobre el empleo de las herramientas virtuales y de los dispositivos móviles en las aulas de secundaria. Bajo este propósito, cabe destacar los estudios de IAB (2017), INJUVE (2017), Ballesteros y Picazo (2018), Smahel et al. (2020) y Díaz, Maquilón y Mirete (2020), entre otros, donde se analizan los usos educativos y los hábitos de comunicación de los estudiantes de secundaria.

Este trabajo parte del marco teórico expuesto para dar respuesta a los siguientes objetivos:

- Determinar las percepciones del profesorado sobre el uso de las redes sociales por parte de los adolescentes y sus potencialidades educativas.

- Examinar los aprendizajes que pueden vehicularse a través del uso de las redes sociales en los niveles de la enseñanza secundaria.

- Establecer los requisitos materiales y formativos del profesorado para incorporar las redes sociales a su tarea educativa.

\section{Materiales y métodos}

El estudio se ha desarrollado a través de una metodología cualitativa. Tal y como señalan Denzin y Lincoln (2012), la investigación cualitativa permite comprender los fenómenos en su contexto natural, interpretarlos a la luz de los significados de las personas implicadas y generar conocimiento a partir de la visión holística que se promueve. De las diversas estrategias de investigación cualitativa que reseñan los autores anteriormente citados, en este estudio se ha optado por la corriente interpretativa (Holstein y Gubrium, 2012), que implica analizar los cómos y los qués de la vida diaria para poder dibujar un panora- 
ma de la realidad que ayude a preguntarse por los porqués y vislumbrar otras alternativas de indagación y de intervención.

Los datos se han obtenido a través de la técnica de la entrevista grupal o grupo de discusión. Se trata de un método muy recomendable cuando se quiere profundizar en ciertos tópicos a través de una conversación abierta con ciertas personas que comparten unas características determinadas de interés para el desarrollo del estudio que se pretende realizar. La decisión de emplear este instrumento se debe a las ventajas que ofrece (Murillo y Mena, 2006): se adapta a realidades complejas, permite a los propios sujetos dar su particular explicación y las relaciones humanas facilitan las opiniones. Para recoger con fidelidad el discurso en su contexto se ha grabado toda la discusión. La transcripción ha sido supervisada por los propios participantes. Previamente se les había informado del objetivo del estudio y del tratamiento confidencial de los datos recogidos.

La entrevista grupal se realizó al finalizar el período escolar, al inicio del mes de julio. Los participantes fueron un equipo de docentes desconocidos entre sí, seleccionados a partir del contacto que se estableció cuando se realizó la aplicación de un cuestionario a estudiantes de secundaria sobre las redes sociales. Se trata de enseñantes muy comprometidos con su tarea docente, que mostraron un verdadero interés por recibir formación sobre el tema. Cumplían la condición de ser profesores de cuarto de ESO y habían hablado con sus alumnos acerca del tema planteado. El conjunto de los miembros representaba distintos polos de una misma realidad: desde el experto en tecnologías de la información o usuario avanzado hasta la profesora inexperta y reticente.

Una vez hechas las presentaciones, agradecida su presencia y explicado el modo en que habría de desarrollarse la sesión, se animó a los participantes a realizar intervenciones libres. El perfil docente de la moderadora facilitó la naturalidad y la espontaneidad para hablar sobre cualquier idea.

Se utilizó un guion de preguntas abiertas que sirvieron de estímulo para favorecer la conversación que, afortunadamente, brotó con espontaneidad y una gran riqueza de ideas. En el guion se establecían tres grandes grupos de interrogantes o cuestiones (figura 1). El primer tema sugerido trataba de deslindar el territorio entre lo conocido y lo desconocido, partiendo de la propia experiencia profesional de los docentes en el entorno virtual y particularmente con las redes sociales. Dentro del segundo apartado se incluían las ventajas que podían aportar en la enseñanza, a nivel de aula y de centro, así como las limitaciones y/o los inconvenientes a tener en cuenta. Por último, se planteó la cuestión acerca de las condiciones necesarias para lograr un uso educativo de las redes sociales. Se preguntaba acerca de la propia trayectoria del profesorado con estas herramientas, de los requisitos para que el uso que hicieran los adolescentes fuera el adecuado y de las demandas formativas que puede requerir su empleo educativo. 
Figura 1. Bloques de temas del grupo de discusión

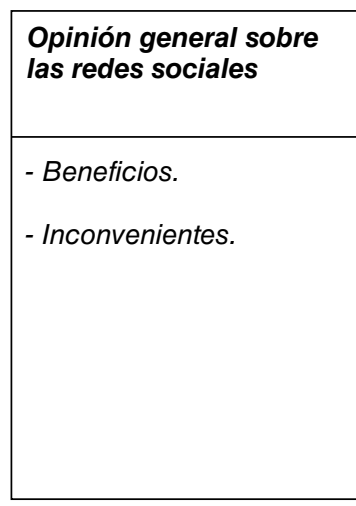

Fuente: elaboración propia.

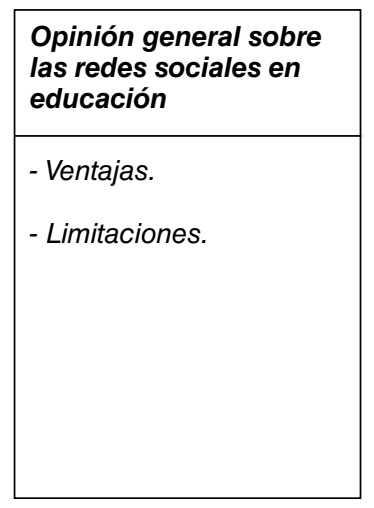

Condiciones para lograr un uso educativo de las redes sociales

- Experiencia docente.

- Requisitos de uso adecuado de las redes por parte de los estudiantes.

A estos tres bloques de cuestiones se les añadieron comentarios sobre la relación entre redes sociales y distintas realidades comúnmente asociadas:

- Redes sociales: privacidad.

- Redes sociales: ciberacoso.

- Redes sociales: adicción.

- Redes sociales: aprendizaje escolar.

- Redes sociales: colaboración.

- Redes sociales: comunicación.

Finalmente se otorgó un tiempo suplementario para que se pudiesen realizar todo tipo de comentarios personales. La duración de la entrevista grupal fue de una hora y treinta minutos.

Se solicitó a los participantes que cubrieran una ficha para registrar algunos datos personales y profesionales de referencia. En esta ficha también se preguntó a los profesores sobre las redes sociales que usaban en su profesión y en la vida personal. Además, se contemplaba un espacio para que expresasen cualquier comentario por escrito sobre la sesión o lo que deseasen compartir con plena libertad.

En la tabla 1 se recopilan las características más significativas del profesorado que participó en la entrevista grupal. Como se puede observar, los intervinientes revelaron distintas procedencias en cuanto al tipo de centro. Se consiguió además un equilibrio entre hombres y mujeres y se procuró, asimismo, que los docentes impartiesen distintas asignaturas, tanto del área de humanidades como del área de ciencias y tecnología.

Se seleccionaron a participantes de diferentes edades y existió además una heterogeneidad en cuanto a características relativas al uso y a la experiencia con Internet y las redes sociales en sus aulas y en sus entornos privados. 
Tabla 1. Características del profesorado participante en la entrevista grupal

\begin{tabular}{clllll}
\hline Clave & \multicolumn{1}{c}{ Centro } & $\begin{array}{c}\text { Asignaturas } \\
\text { impartidas }\end{array}$ & Sexo & Edad & Nivel \\
\hline M1 & Concertado laico & Historia, arte & Femenino & 58 & Nivel bajo \\
\hline M2 & Concertado religioso & Informática & Masculino & 35 & Nivel experto \\
M3 & Concertado laico & Lengua, francés & Masculino & 37 & Nivel medio \\
M4 & IES & Matemáticas & Masculino & 45 & Nivel medio \\
M5 & IES & Matemáticas & Femenino & 57 & Nivel bajo \\
M6 & Concertado religioso & Física & Femenino & 36 & Nivel avanzado \\
\hline
\end{tabular}

Fuente: elaboración propia

Durante el desarrollo de la entrevista grupal se adoptó un rol similar al de los «viajeros» (Flick, 2004) que escuchaban las historias. Y, desde ese mismo momento, mientras asistíamos a los ricos debates que desarrollaron los docentes durante la entrevista grupal, puede decirse que ya se inició el proceso de estudio. El sistema de observación, propiamente dicho, se realizó siguiendo las orientaciones de Miles y Huberman (1994) y bajo la estrategia del análisis de contenido (Goetz y LeCompte, 1988). Concretamente, se combinaron varios procesos: análisis de significado (codificación en categorías, condensación e interpretación hermenéutica); análisis del lenguaje y la pragmática, y uso de sistemas mixtos para la elaboración de metáforas a través de las que sintetizar la visión holística emergente. El proceso fue abierto e inductivo, y emergió de la misma codificación. Una vez segmentado el discurso, marcamos los tópicos que permitían identificar su significado y, en una segunda lectura, procedimos a agrupar ciertos códigos que compartían aspectos comunes para tratar de configurar las categorías y las subcategorías de estudio. En la figura 2 se presenta el sistema de análisis utilizado, especificando las categorías y las subcategorías que emergieron y que se utilizaron para interpretar la entrevista. Se realizó también una codificación transversal para identificar los retos, los

Figura 2. Sistema de categorías y subcategorías para el análisis de la entrevista.

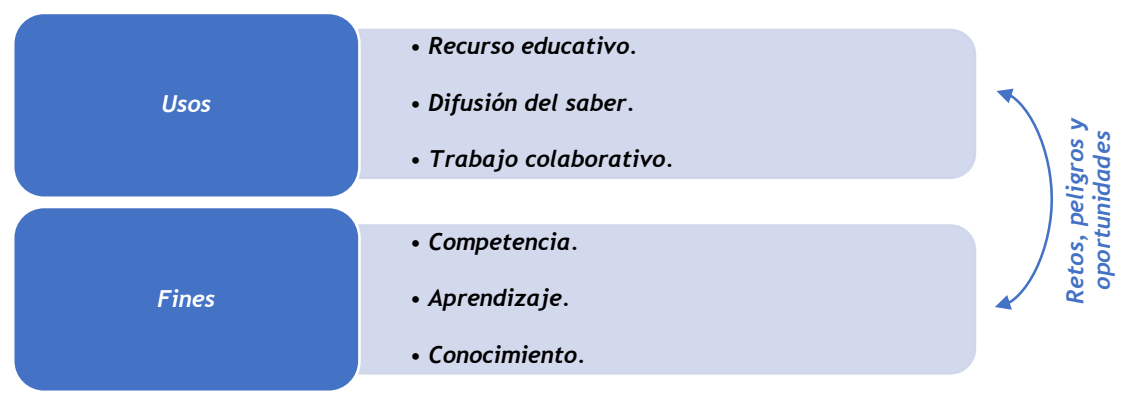

Fuente: elaboración propia. 
peligros y las oportunidades que ofrecían las redes sociales, tanto a nivel general como en el ámbito educativo.

La perspectiva utilizada se dirige al análisis de la dimensión formativa de las redes sociales, y asume este nombre a medio camino entre lo propiamente educativo y lo puramente académico. En este abanico de posibilidades se han marcado dos caminos convergentes: el uso de las redes sociales y la finalidad a la que pueden dar cobertura. En la categoría de uso de las redes sociales, se han incluido dos subcategorías: $a$ ) una función genérica si se considera la posibilidad de la difusión del saber, que haría de las redes sociales un mero canal distribuidor de la información o el conocimiento, y $b$ ) su utilización como recurso educativo (para servir de auxiliar a docentes y alumnos como herramienta de aprendizaje, y también como objeto de aprendizaje en sí mismo, logrando un "uso adecuado" por parte de los adolescentes). La perspectiva formativa entendida ahora no como medio, sino como fin, articula la categoría denominada fines de las redes sociales, que apuntaría a la posibilidad de potenciar nuevas formas de aprendizaje entre los jóvenes, a la ampliación del acceso al conocimiento y otras implicaciones para el desarrollo de las competencias de diversa tipología que se deben fomentar en todo ciudadano y en cualquier profesional.

\section{Resultados}

Tras la codificación y la categorización de los datos se aborda el proceso de interpretación e integración mediante procedimientos de comparación, contraste, ordenación y determinación de vínculos y relaciones (Gibbs, 2012). De esta manera van emergiendo ideas con significados destacados a partir de las que se infieren explicaciones del fenómeno estudiado que ayudan a comprender la realidad y que apuntan a formas de teorización interpretativa. A continuación se presentan los resultados derivados de los análisis desarrollados a partir de la información recopilada en la entrevista grupal y tras el proceso de análisis desarrollado.

Para trazar el informe de resultados de los profesores hemos establecido tres grandes líneas de significado a través de las que se concretan y se proyectan las principales opiniones e ideas expuestas por los participantes:

- Las redes sociales a debate: usos y abusos.

- Aprender con las redes sociales: superar las brechas para favorecer la integración.

- Incorporar las redes sociales al aula: poder, querer y saber.

\subsection{Las redes sociales a debate: usos y abusos}

La cuestión concreta que se planteó en la entrevista grupal acerca de las redes sociales en la educación ha permitido que emergieran las ventajas y las limitaciones de estas, tanto a nivel general como en el ámbito escolar. 
Destaca la estupefacción de los profesores sobre la impavidez de sus alumnos ante el riesgo virtual, sobre todo, frente a los desconocidos. Esta cuestión es fuente de preocupación para los docentes. Valoran como altamente peligroso el uso de perfiles anónimos y el acceso a círculos de relaciones inciertas, aunque sea tan solo como entrenamiento lúdico. Otro hábito de los estudiantes que el profesorado considera negativo es la «vulnerabilidad de la intimidad personal» (M2), puesto que los alumnos están constantemente expuestos en las redes autorrepresentando su estilo de vida a través de sus fotografías, sus vídeos, sus expresiones lingüísticas, etc.: "ponen todo, absolutamente todo. Desde que se levantan hasta que se acuestan [...] No son conscientes de lo expuestos que están» (M6). La intimidad está ligada a la preocupación por la privacidad: «la privacidad ya se ha acabado» (M5). Reiteran la desinhibición que existe al romperse la barrera física y denuncian la incidencia de la falta de buenos modales.

Mención aparte merece el tema del engaño, bien sea como apariencia o como falsedad por parte de «adultos disfrazados» (M2), sobre todo en referencia a la identidad personal, necesaria para actuar con impunidad: «todo vale, eh, parece que el suplantar la identidad en la red no es punible y en la vida real, sí. Ahí es donde tienen confusión» (M2), y del anonimato: «siempre están como escondidos» (M4), «muchas veces no saben quién está detrás de esos contactos» (M2), o bien sea para extorsionar a otros mediante el acoso real o fingido: «ellos conocen el lenguaje y lo utilizan cuando quieren fastidiar a alguien, igual que muchas veces roban un perfil para acosar a alguien haciéndose pasar por otros» (M2). Observan además que se comparten ciertos comportamientos peligrosos respecto a hábitos perjudiciales para la salud, como, por ejemplo: «utilizar las redes sociales para promover bulimia, anorexia [...] dar consejos de cómo hacer si te provocas dolor, quemar calorías» (M3).

Un riesgo advertido por los docentes es la adicción que en gran medida padece el alumnado. Sin embargo, no todos ellos le otorgan una gran importancia, ya que algunos llegan a considerarla un fenómeno universal y no exclusivo de adolescentes, y porque entienden que afecta a un número reducido de jóvenes. En cualquier caso, también afirman que «los chavales a una edad tienen que estar, porque si no son bichos raros» (M4).

Existen reticencias ligadas a la dispersión del alumnado en estos medios o a la inadecuación de las redes para la enseñanza: «las redes sociales las tenemos prohibidas para los alumnos [...] utilizamos más otras herramientas que tenemos para las TIC, pero redes sociales no, ¿eh? Porque no nos parece que sea muy pedagógico y se pierde muchísimo tiempo" (M4).

También citan el uso incontrolado de aparatos y la conexión ininterrumpida como fuente de dispersión frente al estudio. Refuerzan la idea de que mayoritariamente los accesos digitales se vinculan a temas de ocio: «van aprendiendo y para ellos es una herramienta de ocio como otra, pero solo la ven para ocio muchas veces. Ahora sí, si cuando tienen que hacer un trabajo lo aprovechan y le sacan partido» (M2). 


\subsection{Aprender con las redes sociales: superar las brechas para favorecer la integración}

Más allá de las opiniones generales acerca de las precauciones que se deben tomar en torno a las redes sociales, nos encontramos una oposición entre dos polos bien articulados, desde los que se destacan dos conceptos clave: igualdades y desigualdades generadas por las redes sociales.

Las redes se presentan como un instrumento uniformador en cuanto a la identidad personal: «una red social es un sitio donde todos estamos en igualdad de condiciones, ¿no?» (M6), que muestran un estilo de vida semejante y una integración en el grupo de los adolescentes y, por tanto, en la sociedad. No menos importante es la función integradora que tienen las redes respecto a las relaciones con los que permanecen ausentes de las aulas por enfermedad, traslados, programas de idiomas en el extranjero y alumnado emigrante: "cuando algún alumno no puede asistir, puede estar atendido» (M2). La inclusión digital hace posible la educación a distancia, compatible con la presencial, donde se aumenta y se mejora la relación con toda la comunidad educativa (antiguos alumnos, compañeros, padres, etc.), e incluso la relación más técnica con padres (información precisa sobre calificaciones y asistencia): «cuando un padre quiere saber cosas serias del alumno, que son más privadas, ya acude a hablar contigo y lo otro, pues mira, quiere saber la nota, pues ahí te va» (M4). Así pues, la igualdad alude a la posibilidad de aunar al colectivo de jóvenes y profesores entre sí: «es más cómodo comunicar y mandar un mensaje a un compañero desde mi despacho, que tener que andar buscándolo» (M2), y, a su vez, a los profesores como medio de integración en el sector educativo al que pertenecen: "hay que evolucionar, hay que estar con los niños» (M1), así como a sus padres con el centro educativo: «es para lo que vale en este momento, para relacionarnos entre todos» (M5).

Las desigualdades se centran en la persistencia de brechas de distinto tipo y a diversos niveles. La carencia de dispositivos es una de las caras de la brecha digital: «yo estoy en un instituto de la periferia y no todos tienen Internet» (M5), que se suple con recursos de libre acceso en la red: «me da una envidia porque yo no tengo nada de eso en el aula de, en el cole ¿̇no? Entonces yo lo que sí hice fue implementar un sitio donde yo les voy colgando» (M6). El factor económico crea una separación social en las familias y en la titularidad de los centros educativos.

Además, la novedosa igualdad que proporciona la red se opone a la desigualdad entre el docente y el discente: «el profesor tampoco está en igualdad de condiciones con sus alumnos» (M2). La supuesta superioridad técnica del alumnado se enfrenta a la autoridad profesional del profesorado: «es que sabe más que yo... Sí, pero tú tienes más experiencia vital que él y de educación» (M2).

El profesorado se manifiesta a favor de un empeño activo por educar hacia una buena utilización de Internet: «lo que tenemos que hacer es educarles en el uso, que están aprendiendo ellos de forma autónoma. Se creen que todo es 
válido porque es lo que ven. E igual que educamos en otros ámbitos creo que ahí falta que lo integremos en la educación para que lo usen adecuadamente» (M2).

El nuevo rol docente en el aula genera inseguridad: «tengo muchos compañeros muy reacios por miedo» (M2), «muchas veces el mismo profesorado es reacio a los cambios» (M3). Las áreas de conocimiento de las asignaturas dividen la necesidad de utilizar o no las redes sociales en el aula. Así, parece requerirse al profesorado de saberes científicos y técnicos una mayor destreza frente al de humanidades. En conjunto, opinan que no todos los profesionales están preparados para esta tarea. Sin embargo, manifiestan interés por la formación: «sí, me estoy preocupando, pero no sé: es decir, yo creo que hay que incorporarlas, el problema es cómo» (M5).

\subsection{Incorporar las redes sociales al aula: poder, querer y saber}

Una de las condiciones imprescindibles que tendrían que darse para un adecuado uso pedagógico de las redes sociales requiere propiciar un cambio de actitud personal y metodológico. Y estos aspectos entroncan con un elemento que los docentes consideran importante, como es la ética en la educación digital: «cuestiones de ética» (M1). Y se apuesta por introducirla como elemento transversal en todas las asignaturas: «tendría que haber más correlación de unos contenidos con otros. A mí me parecería interesantísimo hoy en día que, en educación para la ciudanía, uno de los temas claves fuese precisamente eso, el uso de las redes sociales» (M6).

A nivel general, valoran muy positivamente su uso, aunque exista cierta perplejidad en su empleo: «me parece interesante, a mí me gustan, me parece que son una manera de conectarse que está bien, que hay que aprovecharla» (M5). Destacan los beneficios en la gestión y el ahorro: «le veo facilidad de comunicaciones [...] Ahorras en muchos sentidos [...] Una vez que lo utilizas realmente le ves todas las ventajas, y ves que te ahorra un montón de trabajo» (M2). Sin embargo, la inversión de tiempo se convierte en un obstáculo: «las redes en clase me comerían un tiempo» (M1) y "tienes que invertir mucho tiempo en buscar» (M2).

En concreto, la motivación es uno de los factores clave para elegirlas: «al final son herramientas motivadoras; muchas veces simplemente como motivación, como herramienta motivacional funciona mejor que hacer un trabajo escrito" (M2). Junto a ello, el aprendizaje competencial que aporta el trabajo colaborativo en redes sociales desarrolla habilidades necesarias para el mundo laboral: «el futuro va por ahí» (M3) y "es que no te queda otra. O te quedas completamente desfasado" (M1).

El modo de aprender cambia y esto provoca modificaciones en el propio trabajo de los docentes, que también debería ser colaborativo y especializado: «que sepa y que tenga tiempo para hacerlo. Como no vengan los materiales de otra forma, lo que sí es posible es hacer un grupo de trabajo de alguien que le guste hacer la tecnología. A alguien que diga "vamos a hacer unidades de esta 
área”. Y alguien que domine las cuestiones informáticas y entre en un grupo de trabajo, se pueda hacer una unidad didáctica» (M2).

Esta demandada conexión profesional conduce a la necesidad de realizar el trabajo en equipo. La solidaridad colaborativa se manifiesta como útil y necesaria entre todos los agentes educativos. En lo que atañe a los profesores entre sí, indican: «estamos desperdiciando las ventajas de las redes sociales que es el compartir. La solidaridad en el compartir conocimientos y trabajo, que sí se hace mucho en la red, pero es que aquí nos faltan infraestructuras y foros adecuados para hacerlo" (M2). En lo que atañe a la comunicación entre los profesores y los padres, los primeros se quejan de los segundos: «los padres no se enteran de lo que no quieren» (M3). La comunicación entre padres y profesores representa un sistema de eficiencia para dar datos como calificaciones o, en el mejor de los casos, información sobre la asistencia a clase a través de la red social del centro. Pero también podría explorarse una mayor implicación de los profesores en su trabajo y en la relación con las familias: «En mi centro funciona lo que son las tutorías de padres, que es una pasada lo bien que funcionan, madre mía, aquí los profesores sí que curran porque solo las respuestas, con toda la cantidad de alumnos en todas las asignaturas, pero se lo curran; está muy bien. Pero también te evitas muchas veces, hay padres que lo agradecen. Porque no pueden ir al centro a entrevistarse con el tutor» (M3).

En síntesis, para que el uso de las redes alcance el éxito educativo es importante gozar de la dotación necesaria de recursos y del apoyo al desarrollo profesional docente: «en mi centro tiene que ser infraestructura y formación» (M2).

\section{Discusión y conclusiones}

A partir de los resultados expuestos y tomando como referencia los objetivos planteados en esta investigación, se pueden extraer las siguientes conclusiones. Con respecto al primero de los objetivos, hay que señalar que a los docentes les asustan los peligros del anonimato, la adicción y la posible inatención por el abuso de las pantallas y, en especial, la fascinación que las redes sociales suscitan en los adolescentes. Reconocen la novedad de las redes sociales, pero a pesar de ello se muestran partidarios de convertir esta realidad digital en una oportunidad para ayudar a su alumnado a crecer y fortalecerse a nivel personal y social a través de un empleo adecuado de las redes sociales, tanto para el ocio como para aprender, ya sea de manera informal o formal. Precisamente, uno de los retos para los docentes es poder incorporar al aula el uso habitual de las redes sociales, que los estudiantes tienen reservado casi en exclusiva para sus actividades de ocio.

Hay coincidencia en valorar positivamente el uso educativo de las redes sociales. Se entiende que los medios digitales pueden facilitar una mejora de la comunicación con las familias y el alumnado. En cuanto a los fines, asumen la competencia digital como algo prioritario en este momento educativo. Emplear adecuadamente las redes sociales contribuye a la mejora de las habilidades necesarias para la preparación personal y profesional de todo ciudada- 
no. En opinión de los docentes, los procesos de enseñanza-aprendizaje no pueden inhibirse de la nueva gestión del conocimiento a través de recursos como los que pueden ofrecer las redes sociales.

Pero también se constata en el profesorado una dificultad real en referencia a la orientación a las familias y a los alumnos (Díaz, Maquilón y Mirete, 2020) en lo que respecta al aprovechamiento de las potencialidades que las redes sociales ofrecen al mundo de la educación (García-Martín y CantónMayo, 2019). En este punto podría afirmarse que el liderazgo docente de los enseñantes está en crisis, como hace años vienen señalando los expertos (Prensky, 2011; Fernández y Shaw, 2020). En muchos casos, la innovación educativa a través de las tecnologías discurre lejana a la experiencia real de los profesores, a sus incertidumbres, preocupaciones y necesidades. Se muestran temerosos frente al cambio profesional que les exigen las nuevas herramientas digitales y desasistidos en la tarea de hacer conscientes a los jóvenes acerca de los riesgos del mundo virtual. De ahí que la necesidad de aumentar la formación y de avanzar hacia un cambio de mentalidad constituyen elementos clave para propiciar la integración de la tecnología en general, y de las redes sociales en particular, a nivel de aula y de centro.

A modo de síntesis, en la tabla 2 se enumeran las ventajas y las desventajas que han perfilado los profesores acerca de las redes sociales en la educación.

Tabla 2. Ventajas y limitaciones de las redes sociales en la educación para el profesorado

\begin{tabular}{|c|c|}
\hline Ventajas & Limitaciones \\
\hline $\begin{array}{l}\text { Fomento de las relaciones con la comunidad } \\
\text { educativa. }\end{array}$ & Fragmentación de la identidad, exhibicionismo. \\
\hline Ahorro de costes y gestiones. & Engaño y falsedad, acoso. \\
\hline $\begin{array}{l}\text { Fomento de nuevas competencias en el alum- } \\
\text { nado. }\end{array}$ & Dispersión del alumnado. \\
\hline $\begin{array}{l}\text { Posibilidad de desarrollar trabajo colaborativo } \\
\text { fuera del aula. }\end{array}$ & $\begin{array}{l}\text { Imagen del profesor perplejo: desigualdad ante } \\
\text { el «alumno experto». }\end{array}$ \\
\hline $\begin{array}{l}\text { Facilitar al profesorado su rol de orientador y } \\
\text { facilitador. }\end{array}$ & Déficit metodológico. \\
\hline Herramientas motivadoras. & Falta de tiempo y formación. \\
\hline $\begin{array}{l}\text { Educación para todos: ausentes, enfermos, } \\
\text { movilidad de estudiantes. }\end{array}$ & Mentalidad anticuada. \\
\hline Innovación: competencia profesional. & \\
\hline
\end{tabular}

Fuente: elaboración propia.

A pesar de las limitaciones de este estudio, tanto a nivel metodológico como por el número de participantes, y sin ánimo de pretender generalizar los resultados, se considera que podrían articularse tres recomendaciones basadas en las demandas expuestas por los docentes. En primer lugar, hay que reconocer que las limitaciones y los riesgos ocupan parte del discurso de los docentes, pero pesan más aún las ventajas. Si bien es cierto que carecen de modelos didácticos para implementar procesos de enseñanza-aprendizaje basados en redes sociales ( $\mathrm{y}$ por ello manifiestan cierta perplejidad acerca de cómo pueden 
llevarlos a la práctica), están a favor de combatir estas dificultades precisamente a través de los nuevos espacios de relación digital con otros docentes.

Confían en su capacidad para afrontar el reto de la integración educativa de las redes sociales, pero la presencia de mentores (López et al., 2018) y el apoyo entre iguales aumentaría su seguridad y su autoeficacia.

Por último, algunos de los requisitos que se exponen como condición de éxito serían los siguientes: la inversión en tiempo, el reconocimiento de los méritos de producción digital, una mayor formación y asesoramiento, un aumento de recursos, etc. Estos exigen el apoyo de la Administración educativa, que tendría que favorecer las redes de colaboración y potenciar el acceso a los recursos formativos.

Los hallazgos de esta investigación permiten documentar la necesidad de realizar más investigaciones de corte cualitativo para comprender en profundidad a los docentes, identificar sus valoraciones y contribuir a mitigar los desvelos y las angustias que les reportan las innovaciones tecnológicas, y en particular las redes sociales, cuando están comprometidos con la mejora de la calidad de la enseñanza y la educación integral de sus estudiantes.

\section{Referencias bibliográficas}

Asterhan, C. y Rosenberg, H. (2015). The promise, reality and dilemmas of secondary school teacher-student interactions in Facebook: The teacher perspective. Computers \& Education, 85, 134-148. $<$ https://doi.org/10.1016/j.compedu.2015.02.003>

Ballesteros, J.C. y Picazo, L. (2018). Las TIC y su influencia en la socialización de los adolescentes. Madrid: Centro Reina Sofía sobre Adolescencia y Juventud.

Cartagena, M. (2016). Uso pedagógico de Facebook y su contribución en la autoeficacia docente. Revista Latinoamericana de Tecnología Educativa, 15(1), 115-129. $<$ https://doi.org/10.17398/1695-288X.15.1.115>

Denzin, N. y Lincoln, Y. (2012). El campo de la investigación cualitativa. Barcelona: Gedisa.

Díaz-López, A.; Maquilón, J. y Mirete, A. (2020). Uso desadaptativo de las TIC en adolescentes: Perfiles, supervisión y estrés tecnológico. Comunicar: Revista Científica Iberoamericana de Comunicación y Educación, 64, 29-38. <https://doi.org/10.3916/C64-2020-03>

Fentry, R.; Boykin, T. y Vickery, K. (2017). Establishing a Framework for Successful Social Network Site Use in the Community College. Community College Journal of Research and Practice, 41(12), 881-896. <https://doi.org/10.1080/10668926.2016.1242440>

Fernández, A. A. y Shaw, G. P. (2020). Academic leadership in a time of crisis: The coronavirus and COVID-19. Journal of Leadership Studies, 14(1), 39-45. $<$ https://doi.org/10.1002/jls.21684>

FLICK, U. (2004). Introducción a la investigación cualitativa. Madrid: Morata.

Fuentes, J.; Albertos, J. y Torrano, F. (2019). Hacia el Mobile-Learning en la escuela: Análisis de factores críticos en el uso de las tablets en centros educativos españoles. Education in the Knowledge Society, 20. <http://dx.doi.org/10.14201/eks2019_20_a3> 
Gamito, R.; Aristizabal, P.; Vizcarra, M. y León, I. (2020). Seguridad y protección digital de la infancia: Retos de la escuela del siglo XXI. Educar, 56(1), 219237. <https://doi.org/10.5565/rev/educar.1113>

García-Martín, S. y Cantón-Mayo, I. (2019). Uso de tecnologías y rendimiento académico en estudiantes adolescentes. Comunicar: Revista Cientifica de Comunicación y Educación, 27(59), 73-81.

Garrido, M.; Busquet, J. y Munté, R. (2016). De las TIC a las TRIC: Estudio sobre el uso de las TIC y la brecha digital entre adultos y adolescentes en España. Anàlisi: Quaderns de Comunicació i Cultura, 54, 44-57. $<$ https://doi.org/10.7238/a.v0i54.2953>

GibBs, G. (2012). El análisis de datos cualitativos en investigación cualitativa. Madrid: Morata.

Goetz, J.P. y LeCompte, M.D. (1988). Etnografía y diseño cualitativo en investigación educativa. Madrid: Morata.

González, M.; Muñoz, P. y Dans, I. (2017). Factors which motivate the use of social networks by students. Psicothema, 29(2), 204-210. <https://doi.org/10.7334/psicothema2016.127>

Greenhow, C. y Burton, L. (2011). Help from my «Friends» Social Capital in the Social Networks Site of Low-Income Students. Journal Educational Computing Research, 45(2), 223-245. $<$ https://doi.org/10.2190/EC.45.2.f>

Holstein, J. y Gubrium, J. (2012). Práctica interpretativa y acción social. En N. Denzin y Y. Lincoln (ed.). El campo de la investigación cualitativa (pp. 228-269). Barcelona: Gedisa.

Hughes, J.E.; Ko, Y.; Lim, M. y Liu, S. (2015). Preservice Teachers' Social Networking Use, Concerns, and Educational Possibilities: Trends from 2008-2012. Journal of Technology and Teacher Education, 23(2), 185-212. Recuperado de <https:// www.learntechlib.org/p/130448/>.

IAB (2017). Estudio anual redes sociales 2017. Recuperado de <http://iabspain.es/wpcontent/uploads/iab_estudioredessociales_2017_vreducida.pdf $>$.

INJUVE. Instituto de LA Juventud (2017). Informe de la juventud 2016. Recuperado de <http://www.injuve.es/observatorio/demografia-e-informacion-general/ informe-juventud-en-espana-2016>.

Jiménez, E.; Garmendia. M. y Casado, M. (2018). Entre selfies y whatsapps: Oportunidades y riesgos para la infancia y la adolescencia conectada. Barcelona: Gedisa.

Junco, R. (2015). Student class standing: Facebook use and academic performance. Journal of Applied Developmental Psychology, 36, 18-29. <https://doi.org/10.1016/j.appdev.2014.11.001>

Kim, J.; Holman, D. y Goodreau, S. (2015). Using Social Network Methods to Test for Assortment of Prosociality among Korean High School Students. PLoS ONE, $10(4), 1-14$. $<$ https://doi.org/10.1371/journal.pone.0125333>

López, S.; Civís, M. y Molina, J.L. (2018). La influencia del capital social en el desarrollo profesional de maestros noveles: Una aproximación con métodos mixtos desde el análisis de redes sociales. Revista de Currículum y Profesorado, 22(2), 89-110. Recuperado de <https://revistaseug.ugr.es/index.php/profesorado/article/ view/7717>. 
MANCA, S. y RANiERI, M. (2015). Implications of social network sites for teaching and learning: Where we are and where we want to go. Education and Information Technologies, 22(2), 605-622. $<$ https://doi.org/10.1007/s10639-015-9429-x>

Miles, M.B. y Huberman, A.M. (1994). Qualitative Data Analysis: An expanded sourcebook. California: SAGE.

Muñoz-Carril, P.C.; Dans-Álvarez-De-Sotomayor, I. y González-SAnMamed, M. (2019). Social Networks and Their Uses in The Field Of Secondary Education. En N. Dey, S. Borah, R. Babo y A. Ashour (eds.). Social Network Analytics. Computational Research Methods and Techniques (pp. 203-226). London: Academic Press. Elsevier Inc. <https://doi.org/10.1016/B978-0-12-815458-8.00011-6>

Murillo, S. y Mena, L. (2006). Detectives y camaleones: el grupo de discusión: Una propuesta para la investigación cualitativa. Madrid: TALASA.

Pablos Pons, J. de; Colás Bravo, P.; Conde Jiménez, J. y Reyes de Cózar, S. (2017). La competencia digital de los estudiantes de educación no universitaria: Variables predictivas. Bordón: Revista de Pedagogía, 69(1), 169-185.

Pedro, L.; Barbosa, C. y Santos, C. (2018). A critical review of mobile learning integration in formal educational contexts. International Journal of Educational Technology in Higher Education, 15(1), 1-10. <https://doi.org/10.1186/s41239-018-0091-4>

Plaza de la Hoz, J. (2018). Ventajas y desventajas del uso adolescente de las TIC: Visión de los estudiantes. Revista Complutense de Educación, 29(2), 491-508. <https://doi.org/10.5209/RCED.53428>

Prensky, M. (2011). Enseñar a nativos digitales. Madrid: SM.

SAID, M. y TAHIR, L. (2013). Towards Identification of Students' Holistic Learning Process through Facebook in Higher Education. Social and Behavioral Sciences, 97, 307-313. <https://doi.org/10.1016/j.sbspro.2013.10.238>

SHIN, W. y Kang, H. (2016). Adolescents' privacy concerns and information disclosure online: The role of parents and the Internet. Computers in Human Behavior, 54, 114-123. <http://doi.org/10.1016/j.chb.2015.07.062>

Smahel, D.; MacHackova, H.; Mascheroni, G.; Dedkova, L.; Staksrud, E.; Olafsson, K.; Livingstone, S. y Hasebrink, U. (2020). EU Kids Online 2020: Survey results from 19 countries. Londres: London School of Economics and Political Science. $<$ https://doi.org/10.21953/lse.47fdeqj01ofo>

Topaloglu, M.; Caldibi, E. y Oge, G. (2016). The scale for the individual and social impact of students' social network use: The validity and reliability studies. Computers in Human Behavior, 61, 350-356. <https://doi.org/10.1016/j.chb.2016.03.036>

Zulkanain, N.; Miskon, S.; Abdullah, N.; Ali, N. y Bahari, M. (2019). Social Network Sites (SNS) Utilization in Learning Landscape - Systematic Literature Review. En Saeed, F.; Gazem, N.; Mohammed, F. y Busalim, A. (eds.). Recent Trends in Data Science and Soft Computing. IRICT 2018. Advances in Intelligent Systems and Computing, 843. Cham. Springer. <https://doi.org/10.1007/978-3-319-99007-1_89> 